\title{
COVID-19, social media use and anxiety: more complex than it might appear?
}

\section{Tim Newton ${ }^{1}$}

\section{A commentary on}

\section{Al-Amad S H, Hussein A.}

Anxiety among dental professionals and its association with their dependency on social media for health information: insights from the COVID-19 pandemic. BMC Psychol 2021; 9: 9.

\begin{abstract}
Design Cross-sectional questionnaire survey of 403 dental healthcare workers.

Sample selection Snowball sampling via social media.

Data analysis Descriptive analysis of sample. Bivariate analysis of the relationship between demographic variables, social media use and anxiety. Binary logistic regression analysis predicting: 1) use of social media; and 2) general anxiety.

Results 1) Social media use was predicted by moderate/severe anxiety level; 2) general anxiety level was predicted by being female and more frequent social media use.

Conclusions The authors conclude that social media reporting of COVID-19 information had adversely affected the psychological wellbeing of dental healthcare workers.
\end{abstract}

\section{Commentary}

The practice of dentistry is accepted to be stressful and to make both physical and mental demands on those who choose to participate in what is both a rewarding and challenging career. The prevalence of anxiety, depression and burnout is acknowledged to be elevated among dental healthcare workers in comparison to the general population. Any major impact upon how members of the dental team work is likely to exacerbate those existing challenges, particularly if the event (as the COVID-19 pandemic has) affects not only work life, but outside work. The article by Al-Amad and Hussein takes as its premise the seemingly plausible notion that feelings of anxiety about the COVID-19 pandemic may be exacerbated by the presentation of information on social media. However, there are both methodological and theoretical issues with their approach. In terms of methodology, there are two main issues: sample selection and the cross-sectional nature of the study. By snowball sampling, the authors have inadvertently selected for social media use - their approach would be unlikely to attract
Practice point

- The mental health and wellbeing of dental healthcare staff is critical to the practice of dentistry. Greater awareness of the wellbeing of all staff should be encouraged, and the influence of external events upon that wellbeing accepted and addressed.

individuals with low levels of engagement with social media. The cross-sectional nature of the study means that we do not know whether there has been a change in the individual's level of anxiety; there is some evidence that the wellbeing of general dental practitioners in the UK was better during the pandemic. ${ }^{1}$ The present study also assumes that all anxiety here is in relation to the pandemic. Of course, there is also the question of the directionality of any relationships. This links to our theoretical understanding of the constructs. Individuals with high levels of anxiety are likely to display hypervigilance - to seek information which may be temporarily reassuring or to gain more information about their 'perceived threat'. Therefore, it would not be unexpected for highly anxious individuals to seek out social media, rather than for social media to generate anxiety. Furthermore, the anxiety detected may be related to other factors such as job security concerns and the health of loved ones. Increased social media use may be a way of seeking information in relation to those concerns.

There can be no doubt that the uncertainty we all face as a result of the pandemic is a likely source of anxiety and that the support of healthcare professionals is vital for a workforce to meet the needs of the population. However, it is incumbent upon us as scientist-practitioners to understand the limitations of our research and qualify our conclusions accordingly.

\section{Author affiliation \\ ${ }^{1}$ King's College London, London, UK. Correspondence: Tim Newton}

\section{References \\ 1. Collin $\mathrm{V}, \mathrm{O}^{\prime}$ Selmo $\mathrm{E}$, Whitehead $\mathrm{P}$. Psychological distress and the perceived impact of the COVID-19 pandemic on UK dentists during a national lockdown. Br Dent J 2021; DOI: $10.1038 / \mathrm{s} 41415-020-2592-5$.}

Evidence-Based Dentistry (2021) 22, 69.

https://doi.org/ 10.1038/s41432-021-0181-3 